\title{
Characterization of Septin Protein Interactions at the Yeast Bud Neck Using a Tripartite Split GFP Detection System
}

\author{
Emily Turnquist ${ }^{1}$, Madison N. Schrock ${ }^{1}$, Megan Halloran ${ }^{1}$, and Gregory C. Finnigan ${ }^{1}$ \\ ${ }^{1}$ Department of Biochemistry and Molecular Biophysics, Kansas State University, 141 Chalmers Hall, \\ Manhattan, KS, USA
}

The septin proteins are a conserved cytoskeletal element found across eukaryotes (including humans) that assemble into filamentous structures in vivo to (i) serve as a barrier between membrane-enclosed compartments, (ii) assist in promoting membrane curvature, and (iii) recruit and bind many non-septin protein targets [1-2]. In Saccharomyces cerevisiae there are five mitotically-expressed septins that are organized into the linear arrangement ("octamer") of Cdc11-Cdc12-Cdc3-Cdc10-Cdc10-Cdc3-Cdc12Cdc11 (with the terminal Cdc11 replaced with an alternate subunit, Shs1) [3]. Previous reports have suggested that nearly 100 other proteins are localized to the division site (bud neck) and may interact with the septins [2]. However, it remains unknown what contingent of proteins physically interacts with the septin structure, and if so, which position(s) along the length of the octamer are occupied. Previously, we demonstrated the use of a tripartite split GFP system to detect protein-protein interactions between nearby septin subunits and four non-septin binding partners [4-5]. Here, we have screened 25 additional candidates using this method by fluorescence microscopy in live cells.

Yeast strains [6] were first manipulated to contain an integrated C-terminal tag consisting of the split GFP $\beta 11$ strand and a plasmid expressing the $\operatorname{GFP}(\beta 1-9)$ fragment. Next, a $\beta 10-\mathrm{Cdc} 3$ strain (also harboring Cdc10-mCherry to mark the bud neck) was chosen as the query for this screen due to its extended $\mathrm{N}$ terminal domain [5]. Finally, strains of the opposite mating types were mated together, diploids selected (SD-LEU-HIS medium), pre-induced in an overnight culture containing raffinose and sucrose, backdiluted, and grown in rich media containing galactose (to activate the GFP( $\beta 1-9)$ construct). Cells were imaged on an inverted Leica DMI6500 fluorescence microscope with a 100x objective lens, Leica DFC340 FX camera, and using the Leica Microsystems software suite and analyzed with ImageJ (NIH).

Diploid yeast pairing interactions between $\mathrm{Cdc} 10 / \mathrm{Cdc} 3$ or $\mathrm{Cdc} 3 / \mathrm{Cdc} 3$ were used as positive controls (Fig. 1A,B) and demonstrated strong GFP signal at the bud neck. Growth in dextrose (inhibiting the GAL1/10 promoter) provided a background level of fluorescence as well as tagging of three highly-expressed, cytosolic proteins (Cdc19, Gpp1, and Tpi1) as previously shown [5]. 25 proteins were chosen that were either (i) large multi-domain proteins (>1,000 residues) or (ii) protein kinases and had previous reports of localization or biochemical interaction with the septins [2,7-8]. Surprisingly, only 1/25 of these proteins, Bud3, displayed a modest, yet statistically significant interaction with the N-terminus of Cdc3 (Fig. $1 \mathrm{~A}, \mathrm{C}, \mathrm{D})$. Testing of the Bud3- $\beta 11$ construct against all five septins revealed a preference for the central subunits, Cdc10 and Cdc3, and co-localized to the external edges of the septin collar at the bud neck (Fig. 1C). Using CRISPR/Cas9, we constructed an N-terminally tagged $\beta 10$-Bud 3 under a constitutive promoter [9] to test against each of the five septins tagged with a $\mathrm{C}$-terminal $\beta 11$ yet no combination yielded an interaction (Fig. 1D). Previous work has suggested that the $\mathrm{C}$-terminal domain of Bud3 may interact with the septins [10]. These data suggest that the split GFP methodology is specific to short-range interactions with precise subdomains of binding partners (tens of nanometers), as there was also no interaction with the C-terminus of Bud4, a protein containing a central septin-binding domain [5]. Future work will focus on identification of smaller septin-binding motifs within large multi-domain proteins [11]. 


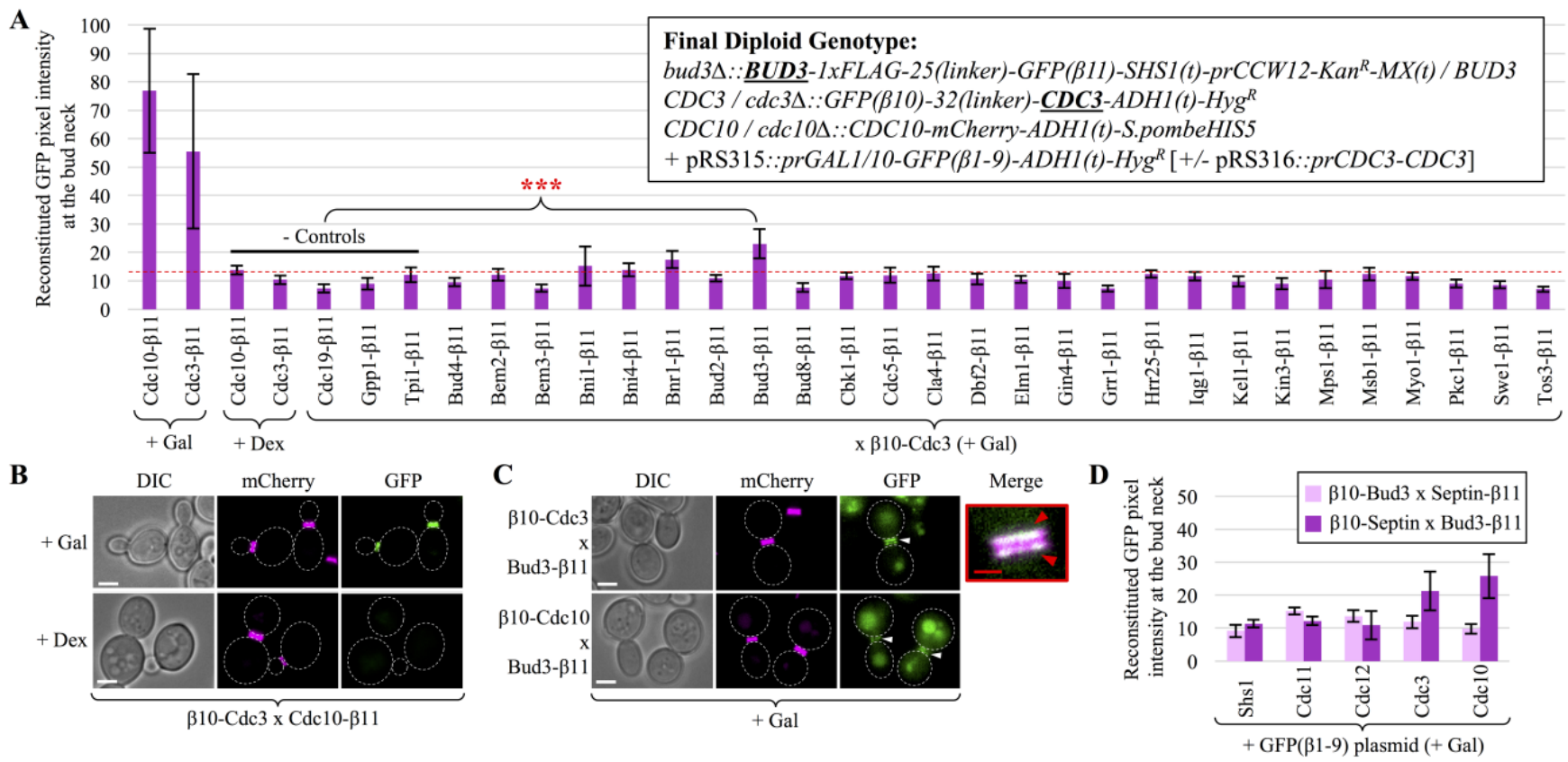

Figure 1. Tripartite split GFP screen for septin-protein interactions at the bud neck reveals the C-terminus of Bud3 binds to the N-termini of Cdc10 and Cdc3. (A) The split GFP system was activated in diploid strains. The level of GFP fluorescence was quantified using ImageJ at the bud neck of cells $(n=10-30)$ displaying a septin collar (Cdc10-mCherry). Error, SD. The genotype of a sample diploid is shown [5]. An unpaired t-test was performed between Bud3 and five controls ( $\mathrm{p}$-value $<0.0001$ for all comparisons). The red dotted line illustrates the average background fluorescence for all negative controls. All samples contained $\beta 10-\mathrm{Cdc} 3$. (B) A positive control (Cdc10- $\beta 11 / \beta 10-\mathrm{Cdc} 3)$ interaction [5] was illustrated with or without galactose induction. Representative cells are shown; all images resized together. White dotted line, cell outline. Scale bar, $3 \mu \mathrm{m}$. (C) Interactions with Bud3- $\beta 11$ are illustrated. White arrows, green fluorescence at the bud neck. Merge, red scale bar is $1 \mu \mathrm{m}$. Red arrows illustrate co-localization between GFP and mCherry signal. (D) Quantification of GFP signal in diploids expressing either Bud3- $\beta 11$ or $\beta 10$ Bud3 against each of the five (tagged) septins following galactose treatment as in (A). Error, SD.

\section{References}

[1] CS Weirich et al, Nat Rev Mol Cell Biol 9 (2008), p. 478-489.

[2] MA McMurray and J Thorner, Cell Div 4 (2009), p. 1-14.

[3] A Bertin et al, Proc Natl Acad Sci USA 105 (2008), p. 8274-8279.

[4] S Cabantous et al, Sci Rep 3 (2013), p. 1-9.

[5] GC Finnigan et al, Mol Biol Cell 27 (2016), p. 2708-2725.

[6] S Ghaemmaghami et al, Nature 425 (2003), p. 737-741.

[7] AM Perez et al, Front Cell Dev Biol 4 (2016), p. 1-12.

[8] WK Huh et al, Nature 425 (2003), p. 686-691.

[9] Roggenkamp et al, Front Microbiol 8 (2017), p. 1-24.

[10] J Guo et al, PLoS One 6 (2011), p. 1-12.

[11] This work was funded by an Institutional Development Award (IDeA) from the National Institute of General Medical Sciences of the National Institute of Health under grant number P20 GM103418 to GCF. This work was supported by a Microscopy Society of America Undergraduate Research Award (summer 2017) to ET and a Kansas State University College of Arts and Sciences Award to MNS. 